\title{
Evaluation of caesarean section practices according to Robson's 10- group classification at a level two maternity ward in Conakry, Guinea
}

\author{
Daniel W. A. Leno ${ }^{1 *}$, Mamoudou E. Bah ${ }^{1}$, Jerry C. Moumbagna ${ }^{1}$, Tamba M. Millimouno², \\ David Lamah ${ }^{1}$, Alexandre Delamou ${ }^{2}$, Telly $\mathrm{Sy}^{1}$
}

\begin{abstract}
${ }^{1}$ Department of Gynecology and Obstetrics, Gamal Abdel Nasser University of Conakry, Conakry, Guinea
${ }^{2}$ Research Unit, National Training and Research Center in Rural Health of Maferinyah, Forecariah, Guinea
\end{abstract}

Received: 16 August 2019

Accepted: 30 September 2019

\section{*Correspondence:}

Dr. Daniel W. A. Leno,

E-mail: danielleno2000@yahoo.fr

Copyright: $\odot$ the author(s), publisher and licensee Medip Academy. This is an open-access article distributed under the terms of the Creative Commons Attribution Non-Commercial License, which permits unrestricted non-commercial use, distribution, and reproduction in any medium, provided the original work is properly cited.

\begin{abstract}
Background: The frequency of caesarean sections (CS) increased dramatically in the world over the last twenty years. The objective of this study was to evaluate caesarean section practices based on Robson classification in an urban referral hospital in Conakry, Guinea

Methods: We conducted a cross-sectional study of 2,266 birthing records collected at the maternity ward of the Coronthie Communal Medical Center in Conakry, from January $1^{\text {st }}$ to December $31^{\text {st }} 2016$. We included in the study all women who had a caesarean section and whose medical records were complete. Robson's classification was used to classify women into 10 groups based on maternal and fetal characteristics. The relative size of each group, its gross caesarean section rate as well as its contribution to overall caesarean section rate and the main caesarean section indications were calculated.

Results: In 2016, 769 caesarean sections were performed out of 2,266 deliveries, corresponding to a hospital section rate of 33.9\%. Groups $5(11.0 \%), 1(4.8 \%)$, and $3(4.3 \%)$ of the Robson classification were the most contributors to registered hospital caesarean section rate. The main indications for caesarean section were uterine scar in group 5 and acute fetal distress in groups 1 and 3.

Conclusions: The systematic reference to the Robson classification could help to identify and avoid the relative indications of the caesarean section in urban Guinea. Besides, increasing induction of labor and strengthening providers' capacities in emergency obstetric and newborn care services could contribute to reduce caesarean section rates in Guinea.
\end{abstract}

Keywords: Evaluation, Caesarean section, Conakry, Guinea, Level two maternity, Robson classification

\section{INTRODUCTION}

The frequency of caesarean sections (CS) increased dramatically in the world over the last twenty years, although the World Health Organization (WHO) recommends that there is no justification for more than 10 to $15 \%$ of deliveries performed by CS in a particular geographic region. ${ }^{1}$ Indeed, high rates of CS constitute a public health problem because of the potential maternal and perinatal risks associated with this intervention, high costs and inequalities of access to obstetric care. ${ }^{1,2}$ In France, after having increased steadily since the 1970 s, the rate of CS seems to be stabilizing at around $20 \% .^{3}$ In Africa, although the population rates of CS are generally very low because of the low financial and geographical accessibility to obstetric services offering this type of intervention, hospital rates are very high, reaching or even exceeding $20 \%$ in some countries to as much as $50 \%$ of deliveries. ${ }^{4}$ In Guinea, studies showed an overall CS rate of $2.7 \%$ and a hospital rate that rose from $21 \%$ in 
2008 to $36 \%$ in $2014 .{ }^{5,6}$ For controlling caesarean section rates, several methods were proposed to analyze CS rates and facilitate geographic or temporal comparisons at the service level. Nowadays, the preference seems to be to analyze CS rates by group with different levels of risk, in order to identify the groups on which efforts must be focused and to propose concrete measures to stabilize or reduce CS rates. The CS classification proposed by Robson in 2001 has advantages and ranks women in 10 categories according to their characteristics and those of the pregnancy. ${ }^{7-9}$ It furthermore makes it possible to compare practices of CS in different maternity wards of the same level per district, region and nationally.

This study aimed at assessing CS practices at a level two maternity ward in Conakry, based on the Robson classification in order to make suggestions for streamlining CS in hospital settings in Guinea.

\section{METHODS}

This study was carried out at the maternity ward of the Communal Medical Center (CMC) of Coronthie, located in the commune of Kaloum in Conakry (the capital city). It is a level two maternity ward (referral hospital). We conducted a cross-sectional study with a sample of 2,266 birthing records from January $1^{\text {st }}$ to December $31^{\text {st }}, 2016$, using a structured questionnaire. We included in this study all women with a gestational age of 28 weeks or more, who had a CS and whose medical records were complete. All CS documented in the medical records were included. The Robson classification was used to classify women into 10 groups, according to maternal and fetal characteristics. ${ }^{7}$ The Robson classification is based on the following characteristics: parity, type of pregnancy (single or multiple), fetal presentation, mode of onset of labor, gestational age, and antecedent of CS (Table 1).
This classification can be made up of 10 or 12 groups according to whether we include both induced labor and planned CS or not, both in primiparous (Groups 2a and b) and in multiparous (Groups $4 \mathrm{a}$ and $4 \mathrm{~b}$ ). Thus, we chose the 10-group classification for its simplicity. The groups 1 to 4 typically correspond to women at low risk of CS since they were constituted of women with term pregnancy and single fetus in cephalic presentation. Then, the groups 5 to 10 correspond to women at high risk of $\mathrm{CS}^{7}$ Data analysis allowed to determine the overall CS rate, to estimate the relative size of each of the 10 groups (number of women in the group divided by the total of women with CS) and to calculate for each group the gross rate of CS (number of women who gave birth by CS divided by the total of women in the group) and the contribution of the group to the overall rate of CS (number of women who gave birth by CS divided by the total number of women). We also classified the leading indications for $\mathrm{CS}$ for the groups that contributed most to overall CS rate.

\section{Statistical analysis}

Data were entered using the Excel software and analyzed using the Epidata analysis software, version 2.2. The confidentiality respect of information collected was maintained by entering and analysing data anonymously. The study was approved by the Guinea National Ethics Committee for Health Research.

\section{RESULTS}

Overall, 2,266 deliveries were realized of which 769 (33.9\%) CS were performed.

Table 1: Robson's 10-group classification. ${ }^{9}$

\begin{tabular}{|ll|}
\hline Group & Definition of obstetric populations \\
\hline 2 & $\begin{array}{l}\text { Nulliparous women with a single pregnancy in cephalic presentation, } \geq 37 \text { weeks gestation in spontaneous labor } \\
\text { induced or were delivered by CS before labor }\end{array}$ \\
\hline 3 & $\begin{array}{l}\text { Multiparous women with a single pregnancy in cephalic presentation, } \geq 37 \text { weeks gestation, without a previous } \\
\text { uterine scar in spontaneous labor }\end{array}$ \\
\hline 4 & $\begin{array}{l}\text { Multiparous women with a single pregnancy in cephalic presentation, without a previous uterine scar, } \geq 37 \\
\text { weeks gestation who either had labor induced or were delivered by CS before labor }\end{array}$ \\
\hline 5 & $\begin{array}{l}\text { All multiparous women with at least one previous uterine scar, a single pregnancy in cephalic presentation, } \geq 37 \\
\text { weeks gestation }\end{array}$ \\
\hline 6 & $\begin{array}{l}\text { All nulliparous women with a single pregnancy in breech presentation } \\
\text { scars multiparous women with a single pregnancy in breech presentation, including women with previous uterine }\end{array}$ \\
\hline 7 & All women with multiple pregnancies including women with previous uterine scars \\
\hline 8 & All women with a single pregnancy in transverse or oblique lie, including women with previous uterine scars \\
\hline 10 & $\begin{array}{l}\text { All women with a single pregnancy in cephalic presentation }<37 \text { weeks gestation, including women with } \\
\text { previous uterine scars }\end{array}$ \\
\hline CS: Caesand
\end{tabular}

CS: Caesarean section. 
Group 5 (multiparas with at least one previous caesarean, single pregnancy cephalic presentation) recorded the highest rate of CS (32.5\%), followed by group 1 (nulliparas with a single pregnancy in cephalic presentation, 37 weeks gestation or more in spontaneous labor) and group 3 (multiparas with a single pregnancy in cephalic presentation, 37 weeks gestation or more, without a previous CS, in spontaneous labor) with respectively $14.1 \%$ and $12.7 \%$.

We noted that the size of group 3 (multiparas with a single pregnancy in cephalic presentation, 37 weeks gestation or more, without a previous CS, in spontaneous labor) was the largest (31.4\%).

Table 2: Distribution of groups by size, rate and contribution to the overall caesarean section rate.

\begin{tabular}{|lllllll|}
\hline Group & $\begin{array}{l}\text { Number of } \\
\text { CS in the } \\
\text { group }(\mathbf{n})\end{array}$ & $\begin{array}{l}\text { Total number } \\
\text { of women in } \\
\text { the group }(\mathrm{N})\end{array}$ & $\begin{array}{l}\text { Group size } \\
(\%)^{1}\end{array}$ & $\begin{array}{l}\text { CS rate in } \\
\text { each group } \\
(\%)^{2}\end{array}$ & $\begin{array}{l}\text { Absolute group } \\
\text { contribution to } \\
\text { overall CS rate of } \\
33.9 \%(\%)^{3}\end{array}$ & $\begin{array}{l}\text { Relative group } \\
\text { contribution to } \\
\text { overall CS rate of } \\
100 \%(\%)^{4}\end{array}$ \\
\hline 1 & 109 & 467 & 20.6 & 23.3 & 4.8 & 14.1 \\
\hline 2 & 69 & 121 & 5.3 & 57.0 & 3.1 & 9.0 \\
\hline 3 & 98 & 712 & 31.4 & 13.8 & 4.3 & 12.7 \\
\hline 4 & 53 & 101 & 4.5 & 52.5 & 2.3 & 3.0 \\
\hline 5 & 250 & 450 & 19.9 & 55.6 & 11.0 & 4.6 \\
\hline 6 & 36 & 65 & 2.9 & 55.4 & 1.6 & 8.1 \\
\hline 7 & 63 & 111 & 4.9 & 56.8 & 2.8 & 4.0 \\
\hline 8 & 30 & 73 & 3.2 & 41.1 & 1.3 & 5.0 \\
\hline 9 & 38 & 62 & 2.7 & 61.3 & 1.7 & 3.0 \\
\hline 10 & 23 & 104 & 4.6 & 22.1 & 1.0 & 100.0 \\
\hline Total & 769 & 2266 & 100.0 & 33.9 & 33.9 & \\
\hline
\end{tabular}

CS: Caesarean section, 1\%: Number (n) of women in the group/total Number $(\mathrm{N})$ of women delivered in the setting $\mathrm{x} 100,2 \%$ : Number (n) of CS in the group/total number $(\mathrm{N})$ of women in the group $\mathrm{x} 100,3 \%$ : Number $(\mathrm{n})$ of $\mathrm{CS}$ in the group/total number $(\mathrm{N})$ of women delivered in the setting x 100, 4\%: Number (n) of CS in the group/total number (N) of CS in the setting x 100.

Table 3: Distribution of the most contributing groups according to indications for caesarean section.

\begin{tabular}{|llll|}
\hline \multicolumn{1}{|c|}{ Groups } & $\mathrm{n}$ & 3 & 5 \\
\hline Indications & $\mathrm{n}(\%)$ & $\mathrm{n}(\%)$ & $\mathrm{n}(\%)$ \\
\hline Acute fetal distress & $57(52.3)$ & $38(38.6)$ & $21(8.4)$ \\
\hline Dystocia of the soft parts & $8(7.3)$ & $13(13.3)$ & $8(3.2)$ \\
\hline Fetal dystocia & $4(3.7)$ & $11(11.2)$ & $8(3.2)$ \\
\hline Bone disorders & $17(15.6)$ & $7(7.1)$ & $45(18.0)$ \\
\hline Hypertensive states of pregnancy & $7(6.4)$ & $9(9.2)$ & $9(3.6)$ \\
\hline Infectious diseases and pregnancy & $1(0.9)$ & $1(1.0)$ & $1(0.4)$ \\
\hline Uterine scar & $1(0.9)$ & $3(3.1)$ & $142(56.8)$ \\
\hline Others* & $14(12.8)$ & $16(16.3)$ & $14(5.6)$ \\
\hline
\end{tabular}

*Others: infertility, history of stillbirth, suitability.

Concerning the contribution (absolute contribution) to overall CS rate of $33.9 \%$, our findings showed that multiparous women with at least one previous CS, a single pregnancy in cephalic presentation (Group 5) were the main contributor to overall $\mathrm{CS}$ rate with $11.0 \%$ followed by groups $1(4.8 \%)$ and $3(4.3 \%)$ (Table 2$)$.

The main indications for CS in the groups contributing most to overall rate of CS were uterine scar in Group 5 $(56.8 \%)$ and acute fetal distress in groups $1(52.3 \%)$ and $3(38.6 \%)$ (Table 3).

\section{DISCUSSION}

This study found an overall rate of CS (33.9\%) close to that of national hospital data $(36.0 \%)$ in Guinea. ${ }^{5}$ However, our findings are higher than those observed in level 2 maternity clinics in Senegal and France, with respectively $18.2 \%$ and $21.1 \%$ of CS rates. ${ }^{10,11}$ This could be explained, on the one hand, by the increase of obstetric care at Coronthie CMC since 2015, due to the closure of the maternity ward of Donka national hospital for renovation and which required the redeployment of its team at Coronthie CMC and on the other hand, by the 
systematization of CS in cases of breech presentation and uterine scar, financial motivation and personal convenience, without forgetting the variability of the definitions of dystocia and the resulting practices. According to the WHO, rates of caesarean deliveries in health facilities vary considerably depending on the composition of the obstetric populations they care for, health facilities capacities and resources, and clinical management protocols of health facilities. Because of these differences, there is no ideal rate at the hospital level. ${ }^{1}$

The predominance of group 5 in our study (multiparas with at least one previous CS, a single pregnancy in cephalic presentation) could be explained by the strengthening of maternity care at Coronthie CMC allowing the effective management of obstetric emergencies from Conakry and neighboring cities. Our results are contrary to that of a study conducted in France which, in a different context, found that women at low risk of $\mathrm{CS}$ of Group 3 (multiparous with a single pregnancy in cephalic presentation, $\geq 37$ weeks gestation, no history of $\mathrm{CS}$, spontaneous labor) were the more represented. ${ }^{11}$

Regarding the size of the group, our findings are similar for groups 3 and 1 to those of that study in France with, in contrast, higher rates, $31.7 \%$ and $26.6 \%$, respectively. ${ }^{11}$

Group 5, with an absolute overall contribution of $11.0 \%$, weighs heavily in the overall rate of CS in our study. Our results are consistent with literature data this suggests that a history of CS is one of the leading causes of CS..$^{8,10,12-14}$ However, some studies reported a success rate of vaginal deliveries between $60-90 \%$ after a satisfactory CS. ${ }^{15,16}$

Thus, the management of the vaginal delivery appears as one of the most effective mechanisms for reducing the progression of the overall CS rate. Indeed, providers number and capacity building in providing quality obstetric care are required especially in sub-Saharan African settings. In addition, two groups (1 and 3) of low risk of CS populations were among the top three contributors to the overall CS rate. These findings could be explained by obstetric referrals due to, among others, the poor quality of antenatal follow-up, the poor access of the population to health facilities, the proliferation of unconventional birthing centers and care structures (underqualified staff and insufficient equipment), the insufficiency of qualified health care providers in general.

According to Main et al, the CS rate in group 1 can be considered as an indicator of the quality of obstetric care in a maternity ward. ${ }^{17}$ Improving provider's capacities and people's access in and to emergency obstetric and neonatal care could help to effectively reduce hospital CS. So, if women have better access to the seven signal functions in their health center, unnecessary referrals could be reduced and those women who need a CS could benefit from a referral. A study carried out in Senegal revealed similar findings regarding the group contribution, but with Group 1 being the largest contributor with $34.2 \% .{ }^{10}$ On the other hand, our results are consistent to those found in a study conducted in France where Group 5 was the largest contributor (6.0\%) to the overall CS rate. ${ }^{11}$ However, the overall rate (33.9\%) of CS we found is much higher than that found $(12.7 \%)$ in level 2 maternity wards in France. ${ }^{11}$

These findings could be explained by different obstetric practices with regard to the management of women with scarred uterus and obstetric emergencies.

In our study, uterine scar was the dominant indication for caesarean section in group $5(56.8 \%)$. Our results are closer to national data, which showed $49.0 \%$ of indications for avoiding risks and uncertainties as the most common. ${ }^{6}$ Repeated caesarean sections, for fear of complications such as uterine rupture, is recognized as the major cause of the increase of CS rates. To counteract this tendency, a uterine test might be performed after the evaluation of obstetric status and other absolute indications for CS (transverse presentation, placenta previa, forehead (front) presentation, corporal scar, and antecedent of at least two previous CS).

The practice of labor induction on a single-scar uterus should be encouraged in our context with a closer monitoring to control the risk of uterine rupture although this is very minimal. In fact, the labor induction on the scarred uterus increases the risk of uterine rupture, which can be estimated at $1 \%$ when oxytocin is used and $2 \%$ when prostaglandins are used. ${ }^{18}$

In this context, the National College of French Gynecologists and Obstetricians recommends the prudent use of oxytocin and intra-cervical or extra-amniotic balloon for the artificial labor induction in this indication, hence the interest of training obstetricians on the techniques of artificial labor induction and the regular monitoring of induced labor. ${ }^{19}$ The regular practice of artificial labor induction for single-scar uterus while conforming to required clinic protocols could help to reduce unnecessary hospital CS rates.

For the groups 1 and 3, fetal distress was the dominant indication with rates of $52.3 \%$ and $38.6 \%$, respectively. This could be explained by the fact that it was about women belonging to groups with low-risk of CS, most often referred from peripheral health facilities after having made a long stay and received in an obstetric emergency context. It is in this context of obstetric emergency that the acute fetal distress is diagnosed clinically (alteration of the fetal heart rate with or without the greenish or meconial aspect of the amniotic fluid). In Guinea, CS are most often performed urgently in $84.9 \%$ of cases. ${ }^{6}$ Therefore, minimizing obstetric emergencies and the use of caesarean sections on "virgin" uterus in low-risk populations (Groups 1 and 3) should be a constant concern of providers. According to Haydar and 
Coll, the reduction in CS in the nulliparous (Groups 1 and 2) would directly influence the number of CS in women of Group 5 (women with previous CS) in the future, hence the interest of improving emergency obstetric and newborn care at the primary level of care through information and no cost obstetric care. ${ }^{12}$

Our study has limitations including the small sample size and the mono-centric character since data concern only one maternity ward. Thus, our findings cannot be generalized to the whole Guinean population because of the diversity of practices in the country. However, we diffuse our results to encourage other maternity wards to use Robson classification to analyze their CS practices and compare them with those of other maternity wards nationally and internationally.

\section{CONCLUSION}

This study revealed that the most contributing groups to the overall CS rate were groups 5, 1 and 3. The main indications for CS were uterine scar and acute fetal distress. Hence actions such as popularizing the practice of the artificial labor induction on the single-scar uterus, increasing induction of labor and strengthening providers' capacities in emergency obstetric and newborn care services could contribute to reduce caesarean section rates in Guinea.

\section{Funding: No funding sources}

Conflict of interest: None declared

Ethical approval: The study was approved by the Institutional Ethics Committee

\section{REFERENCES}

1. Vogel JP, Betrâ̂n AP, Vindevoghel N, Souza JP, Torloni MR, Zhang J, et al. Use of the Robson classification to assess caesarean section trends in 21 countries: a secondary analysis of two WHO multicountry surveys. Lancet GlobHealth. 2015;3:260-70.

2. Betran AP, Ye J, Moller AB, Zhang J, Gulmezoglu $\mathrm{AM}$, Torloni MR. The increasing trend in caesarean section rates: global, regional and national estimates: 1990 \pm 2014 . PloS One. 2016;11:e0148343.

3. Blondel B, Lelong N, Kermarrec M, Goffinet F. Trends in perinatal health in France between 1995 and 2010: Results from the National Perinatal Surveys. J Gynecol Obstet Biol Reprod. 2012;41:151-66.

4. Cissé CT, Ngom PM, Guissé A. Reflections on the evolution of caesarean section rates in Africa: example of the Dakar University Hospital Center between 1925 and 2001. Gynecol Obstet Fertil. 2004;32:210-7.

5. Ministry of Health. Needs assessment of emergency obstetric and newborn care in republic of Guinea. Final report. Ministry of Health; 2013.
6. Keita N, Diallo FD, Gandaho E, Leno DWA. Caesarean section in Africa: Example from Guinea. French national college of obstetricians and gynecologists, Fourth part. Gynecology-obstetrics of the world, 38th national days, Paris; 2014:337-346.

7. Robson MS. Can we reduce the caesarean section rate? Best Pract Res Clin Obstet Gynaecol. 200;15:179-94.

8. Robson M, Hartigan L, Murphy M. Methods of achieving and maintaining an appropriate caesarean section rate. Best Pract Res Clin Obstet Gynaecol. 2013;27:297-308.

9. World Health Organization. Robson classification: Implementation manual. 2017. Available at: https://apps.who.int/iris/bitstream/handle/10665/259 512/9789241513197-eng.pdf;jsessionid=8763FB EBBD12B2CADC7FC4CE085500B1? sequence $=1$. Accessed on $1^{\text {st }}$ August 2019.

10. Mbaye M, Gueye M, Gueye MDN, Niang NKS, Moreau JC. Analysis of cesarean section rate according to Robson's classification in an urban health centre in Senegal. Int J Reprod Contracept Obstet Gynecol. 2015;4:1100-2.

11. Le Ray C, Prunet C, Deneux-Tharaux C, Goffinet F, Blondel B. Robson classification: A tool for assessing caesarean section practices in France. J Gynecol Obstet Biol Reprod. 2015;44:605-13.

12. Haydar A, Vial Y, Baud D, Desseauve D. Evolution of the caesarean sections rate in a Swiss university maternity hospital according to Robson classification. Rev Med Suisse. 2017;13:1846-51.

13. Nakamura-Pereira M, Carmo LM, Esteves-Pereira AP, Domingues RMSM, Alves Torres J, Bastos Dias MA et al. Use of Robson classification to assess cesarean section rate in Brazil: the role of source of payment for childbirth. Repro Health. 2016;13(Suppl. 3):245-56.

14. Mbungu MR, Ntela MJ, Kahindo MP. Frequency of caesarean sections according to Robson classification in 3 maternity wards in the city of Kinshasa, Democratic Republic of Congo. Ann Afr Méd. 2017;10(2):2535-44.

15. Studsgaard A, Skorstengaard M, Glavind J, Hvidman L, Uldbjerg N. Trial of labor compared to repeat cesarean section in women with no other risk factors than a prior cesarean delivery. Acta Obstet Gynecol Scand. 2013;92:1256-63.

16. Guise JM, Berlin M, Mc Donagh M. Safety of vaginal birth after cesarean: a systematic review. Obstet Gynecol. 2004;103:420-9.

17. Main EK, Moore D, Farrell B. Is there a useful cesarean birth measure? Assessment of the nulliparous term singleton vertex cesarean birth rate as a tool for obstetric quality improvement. Am J Obstet Gynecol. 2006;194:1644-51.

18. Duruelle P, Lepage J, Depret S, Clouqueur E. Mode of labour induction and labour conducting in case of uterine scar. J Gynecol Obst Biol Reprod. 2012;41(8):788-802. 
19. Delivery in women with previous cesarean section or other uterine surgery: guidelines for clinical practice - text of the guidelines (short text). J Gynecol Obstet Biol Reprod (Paris). 2012;41:824-30.
Cite this article as: Leno DWA, Bah ME,

Moumbagna JC, Millimouno TM, Lamah D, Delamou

A, et al. Evaluation of caesarean section practices according to Robson's 10-group classification at a level two maternity ward in Conakry, Guinea. Int J Reprod Contracept Obstet Gynecol 2019;8:4468-73. 